\title{
Supporting information: Tailor: non-parametric and rapid score calibration method for database search-based peptide identification in shotgun proteomics
}

\author{
Pavel Sulimov ${ }^{1}$ and Attila Kertész-Farkas ${ }^{1}$ \\ ${ }^{1}$ Department of Data Analysis and Artificial Intelligence, Faculty of Computer Science, \\ National Research University Higher School of Economics (HSE), 3 Kochnovskiy Proezd, \\ Moscow, 125319, Russian Federation
}

February 28, 2020

\section{Contact information}

Attila Kertesz-Farkas, e-mail: akerteszfarkas@hse.ru

\section{Content}

- S-1 Database search program parameters (This document).

This section contains additional information the database-searching programs and the parameters we used in our experiments.

- S-2 Supplementary figures (This document).

This section contains additional plots about score distributions referred from the main text.

- Supplementary File 1 (scripts.zip): The scripts zipped used to run all methods in our tests. The files contain the scripts we used to run our experiments with all the parameters.

- Supplementary File 2 (HSPP2A.zip): The HSPP2A dataset zipped. This file contains the HSPP2A tandem mass spectrometry file. It is provided here because it is not available in the original repository.

- Supplementary File 3 (Fasta.zip): The fasta files zipped, used in our performance. The files contains the predigested target and decoy peptides we used in our experiments.

\section{Supplementary Note S1: Database search program parameters}

The scripts we used to run the following methods along with their parameterization to perform our experiments can be found among the supplementary files.

Tide-search. The tide-search program [1] from Crux [2] version 3.2.8aa66a2 was downloaded and used in Linux to execute XCorr and Res-ev score functions and their corresponding exact p-value (XPV) calculation methods. We note that the raw XCorr score function and the XCorr score function for the XPV algorithms are carried out with different code, and they are also slightly different. The raw XCorr considers only singly charged fragment ions for precursors of charge $1+$ or $2+$; however, it considers singly and doubly charged fragment ions for a precursor with a charge state larger than 2+ [1]. In contrast, the XCorr score function in the XVP calculation considers the fragment ions of charge $n-1$ for a precursor ion if charge $n>1$ [3]. This discrepancy can result in different discrimination power for the score functions making the comparison 
of their calibration power more difficult. Therefore, we adjusted the source code of the XPV method to raw XCorr score function so that they become equivalent. A patch file performing this code update is available in the supplementary materials.

The tide-search program was run with the following parameters: min-peaks $=10$, use- neutral- loss-peaks $=F$, concat $=\mathrm{T}$, max-precursor-charge $=9$. The precursor-window, precursor- - window- type, and mz-bin-width were specified with respect to the input spectrum dataset. The p-values for the XCorr scores were obtained using the exact-p-value $=\mathrm{T}$ parameter, and the $\mathrm{p}$-values obtained were adjusted to the multiple hypothesis testing using the Šidák correction [4]. The Res-ev score function [5] was used for high-resolution MS2 data (HumVar, Malaria, iPRG).

Tide-index generated the preprocessed peptide index files with the following parameters: decoy-format= none, enzyme $=$ custom-enzyme, custom-enzyme $=[Z] \mid\{Z\}$ and missed-cleavages $=0$. Modifications were specified for the given taxon. All other parameters were used with their default values.

X!Tandem. The X!Tandem version of 2015 was used with the following parameter settings: cleavage site $=[Z] \mid\{Z\}$, refine $=$ No, minimum peaks $=10$, total peaks=50, output, results=all. Note that, the $\mathrm{X}$ !Tandem defines the fragment ion peak match tolerance as $|p-m|<t$; therefore, $\mathrm{mz}$-bin-width $=1.0005079$ would be equivalent to fragment monoisotopic mass error=0.5 Da, which leads to roughly the same spectrum discretization step. For high-resolution data, fragment monoisotopic mass error=0.01 Da was specified, which is equivalent to $\mathrm{mz}$-bin-width $=0.02$. Other dataset-specific parameters were specified accordingly. The X!Tandem program uses the HyperScore scoring function defined as HyperScore $(s, t)=$ $N_{b} ! N_{y} ! M(s, t)$, where $N_{b}$ ! and $N_{y}$ ! denotes the factorial of the number of matching $b$ and $y$ ions in the theoretical spectrum.

The Tailor score calibration of the HyperScore scores was executed on the output of the X!Tandem program (.xml file), and it was implemented as a Python script. We note that the experimental spectra, that did not have at least 30 candidate peptides were excluded from our analysis. We also note that X!Tandem does not report the PSMs of the candidate peptides individually for every experimental spectrum, it reports only the histogram of the empirical cumulative survival distribution of the HyperScore scores, and all relevant information needed for Tailor, such as the number of the candidate peptides and the raw HyperScore scores, was extracted from this histogram.

OMSSA. The OMSSA program version 2.1.9 was downloaded from ftp://ftp.ncbi.nlm.nih.gov/pub/ lewisg/omssa/2.1.9/. For this program, the fasta files were preprocessed with the makeblastdb program of the BLAST toolbox version 2.9.0+ with the parameter -dbtype prot. The omssacl program was run with the following parameters: $-i$ 1,4 ( $b$ and $y$ ions used), -mnm (disabling protein N-terminal methionine cleavage), - $\mathrm{mf} 3$ (static carbamidomethylation of cysteine), -e 11 (disabling in silico digestion) -he 99999999 (maximum e-value), -hl 9999 (retain top 9999 hits), -zcc 1 (believe precursor charge), -tem 0 -tom 0 (monoisotopic precursor and fragment ions), -tez 1 (linear charge dependency of precursor charge), texttt-zh 9 (max charge state), -v 0 (number of missed cleavages 0 ), and -oc (csv output). The precursor ion (-te, -teppm) and the fragment ion tolerance (to) parameters were specified with respect to the input spectrum dataset. All other parameters remained at their default value.

We note that we did not implement Tailor inside the OMSSA program, but as a Python script, which post-processes the comma-separated csv output of OMSSA. OMSSA was parameterized to report all PSMs corresponding to all candidate peptides for all experimental spectra so that the Tailor method could extract the information needed. We also note that the experimental spectra that have fewer than 30 candidate peptides were excluded from our analysis.

MSGF + The MFGs $+v 2018.01 .30$ was with the following parameters: $t d a=0$, addFeatures $=1$, ignoreMetCleavage $=1$, $\mathrm{e}=9 \mathrm{n}=200$, and $\mathrm{ti}=0,0$. To run MSGF + with high-resoltion ms2 information the following parameters were set inst=1 $\mathrm{m}=3$ for Plasmodium data, and inst=1 $\mathrm{m}=3$ for the Human Variation data, respectively. For low resolution ms2 settings these parameters remained on default values. The resulted output (mzid) was post-processed with a Python script to apply Tailor score calibration. The Python script is available in the supplementary files. We note that, we were not able to run MSGF+ on the HumVar data, because MSGF+ incorrectly recognized it as a profile spectrum data file, and on the Yeast dataset, because MSGF + crashed on it possibly due to out of memory error. 

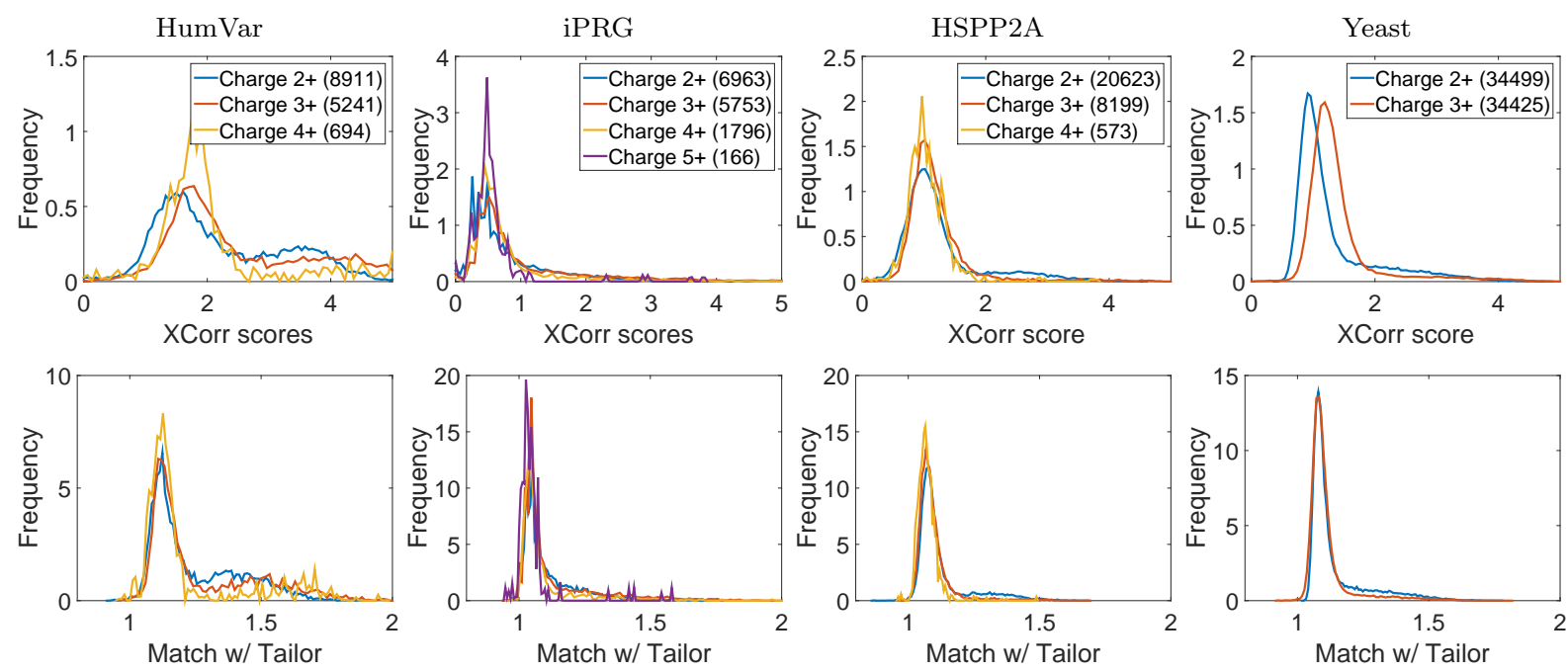

Figure S1: Score distributions of the XCorr (top row) and the Tailor (bottom row) methods with respect to precursor ion charge states. The columns correspond to different datasets and share a figure legend. The distributions were smoothed with the Gaussian kernel. The PSM scoring was carried out via concatenated target-decoy search.

\section{Supplementary Note S2: Supplementary figures}



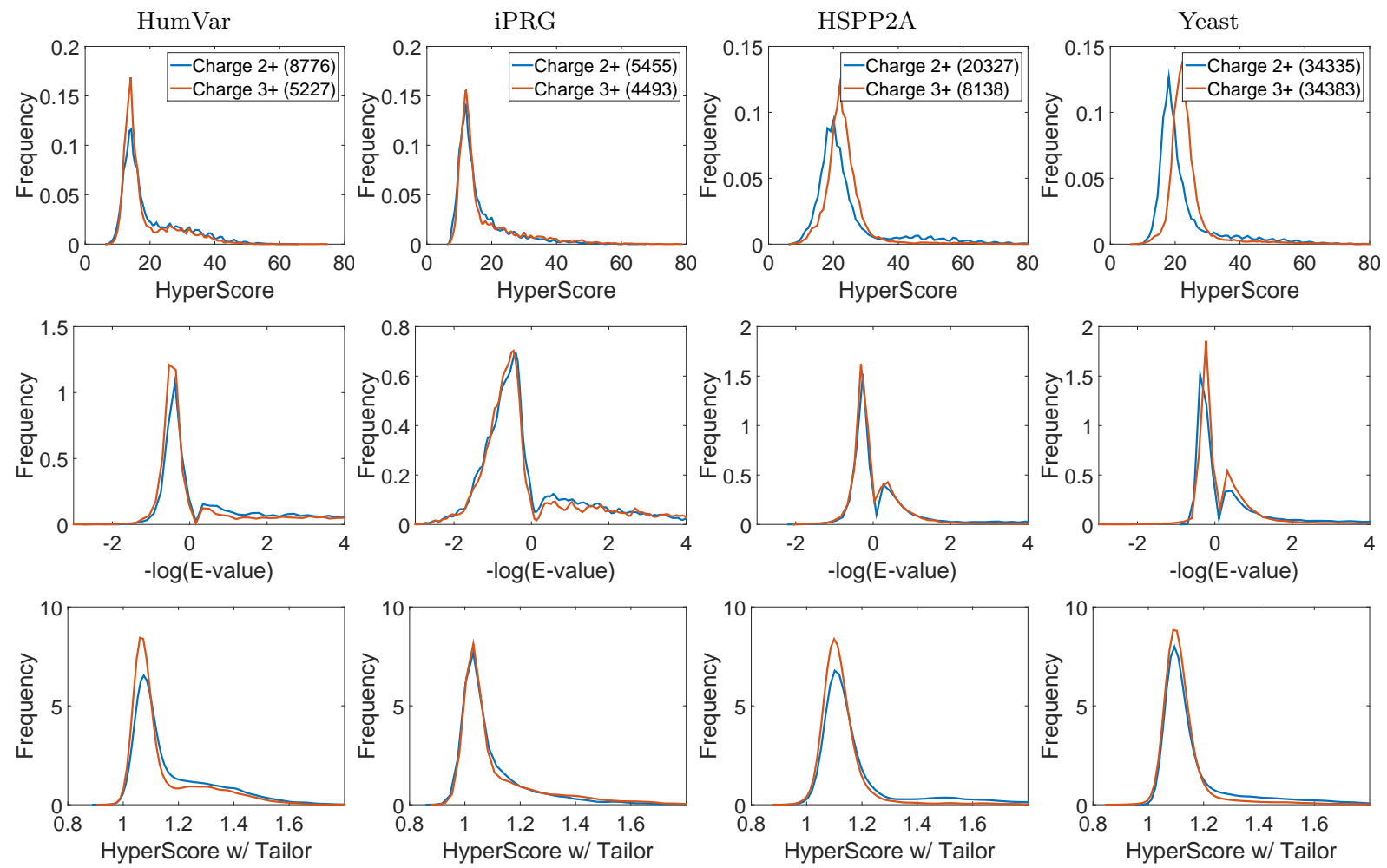

Figure S2: Score distributions of the HyperScore (top row), log E-value (middle), and the HyperScore calibrated with Tailor (bottom row) methods with respect to precursor ion charge states. The columns correspond to different datasets and share figure legend. The distributions were smoothed with the Gaussian kernel. The PSM scoring was carried out via concatenated target-decoy search.
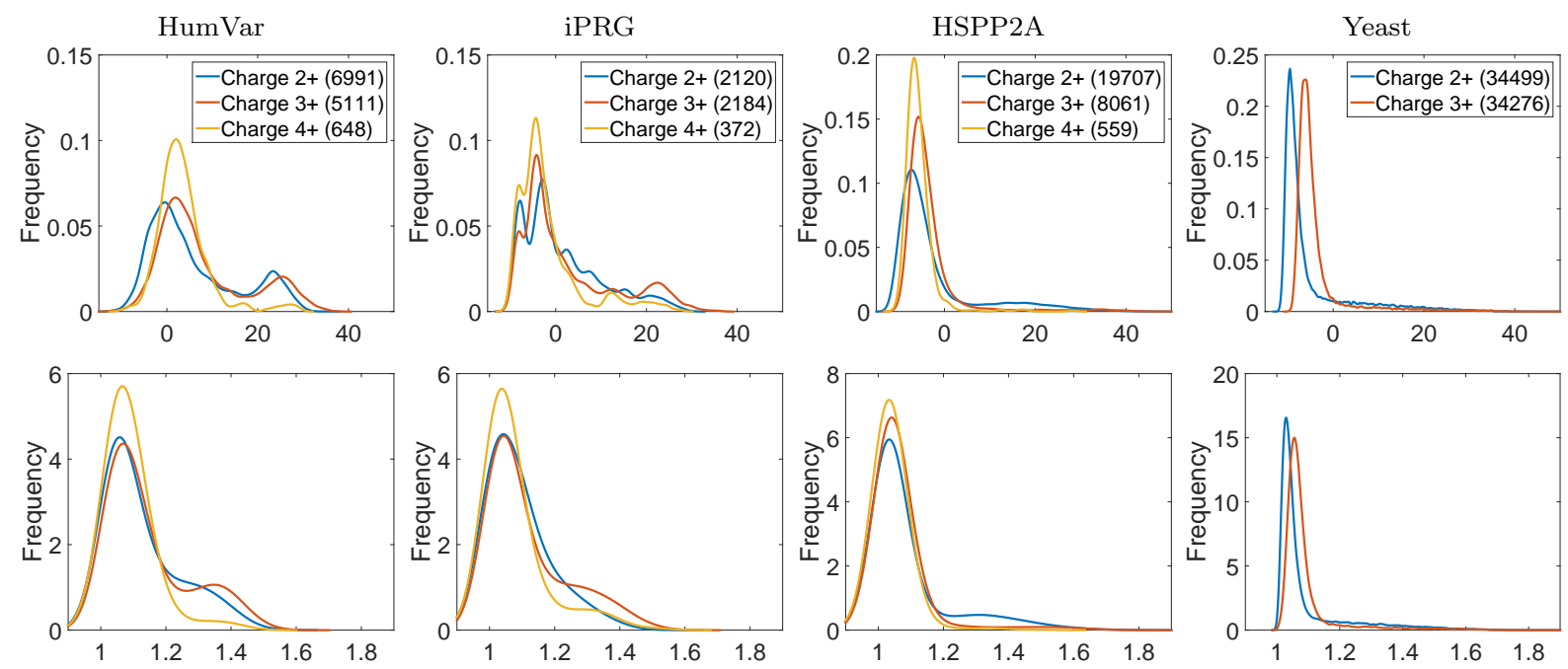

Figure S3: Score distributions of the top-scoring PSMs with respect to the charge state of the precursor ions. Each column corresponds to a dataset, plots in the top row correspond to OMSSA's p-value, and plots in the bottom row corresponds to the Tailor method. The distributions were obtained with kernel smoothing using the Gaussian smoother. The PSM scoring was carried out via concatenated target-decoy search. 
A) Yeast

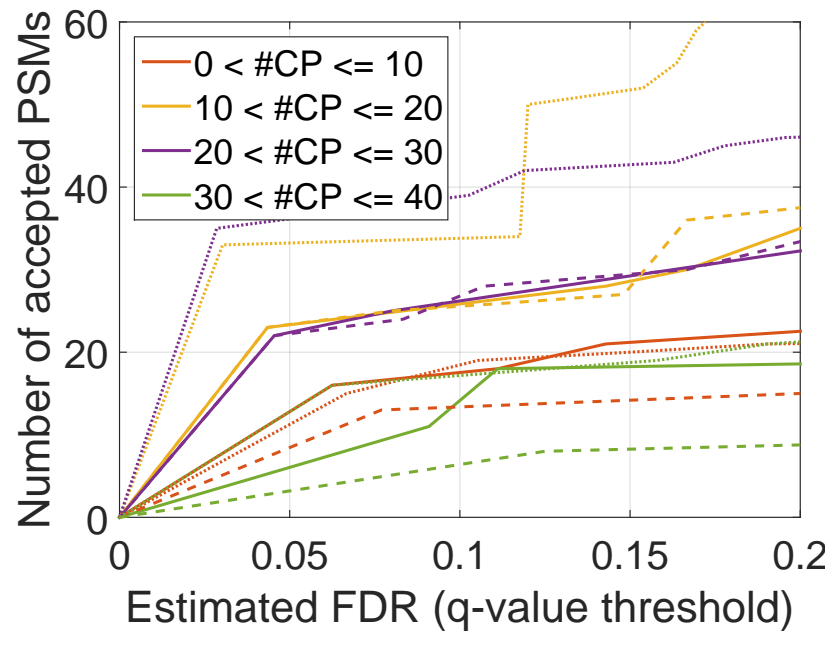

Figure S4: The XCorr, Match score with Tailor calibration, and XPV were re-run on the Yeast datasets using a narrow precursor tolerance window: $10 \mathrm{ppm}$. This resulted in 22,714 annotated spectrum (out of 69,705 ) of which (a) 14,590 had candidate peptides less than or equal to 10, (b) 5,243 had candidate peptides more than 10, but less than or equal to 20, (c) 1,926 had candidate peptides more than 20, but less than or equal to 30, and (d) 668 had candidate peptides more than 30, but less than or equal to 40 . 364 spectrum annotations had more than 40 candidate peptides and the largest number of candidate peptides were 90. The average number of candidate peptides was 10.45 .

\section{References}

[1] Benjamin J Diament and William Stafford Noble. Faster sequest searching for peptide identification from tandem mass spectra. Journal of Proteome Research, 10(9):3871-3879, 2011.

[2] Sean McIlwain, Kaipo Tamura, Attila Kertesz-Farkas, Charles E Grant, Benjamin Diament, Barbara Frewen, J Jeffry Howbert, Michael R Hoopmann, Lukas Käll, Jimmy K Eng, et al. Crux: rapid open source protein tandem mass spectrometry analysis. Journal of Proteome Research, 13(10):4488-4491, 2014.

[3] J Jeffry Howbert and William Stafford Noble. Computing exact p-values for a cross-correlation shotgun proteomics score function. Molecular $\&$ Cellular Proteomics, 13(9):2467-2479, 2014.

[4] Zbyněk Šidák. Rectangular confidence regions for the means of multivariate normal distributions. Journal of the American Statistical Association, 62(318):626-633, 1967.

[5] Andy Lin, J Jeffry Howbert, and William Stafford Noble. Combining high-resolution and exact calibration to boost statistical power: A well-calibrated score function for high-resolution ms2 data. Journal of Proteome Research, 17(11):3644-3656, 2018. 